\title{
MHD Flow of Micropolar Fluid in a Rectangular Duct with Hall and lon Slip Effects
}

dsrinivasacharya@yahoo.com, dsc@nitw.ac.in National Institute of Technology Department of Mathematics Warangal, India

\author{
Mekonnen Shiferaw \\ mekonnenjan2004@yahoo.com \\ National Institute of Technology \\ Department of Mathematics \\ Warangal, A.P. India \\ Permanent address : ArbaMinch University \\ Department of Mathematics \\ PO Box 72 \\ Arba Minch,Ethiopia
}

The steady flow of incompressible and electrically conducting micropolar fluid flow through a rectangular channel is considered taking Hall and ionic effects into consideration. An external uniform magnetic field is applied which is directed arbitrary in a plane perpendicular to the flow direction. The governing partial differential equations are solved numerically using finite difference method, and the effects of micropolar parameters, magnetic parameter, Hall parameter and ion slip parameter on the velocity and microrotation are discussed.

Keywords: magnetohydrodynamics, hall and ion slip effects, micropolar fluid, rectangular duct

\section{Introduction}

The increasing number of technical applications using magnetohydrodynamic (MHD) effects has made it desirable to extend many of the available hydrodynamic solutions to include the effects of magnetic fields for those cases when the fluid is electrically conducting. Several investigators have studied the fluid flow between parallel plates, through a circular pipe and through a rectangular duct in the presence of a transverse magnetic field. Hunt (1965) analyzed the laminar motion of a conducting liquid in a rectangular duct under a uniform transverse magnetic field and obtained exact solutions for (i) perfectly conducting walls perpendicular to the field and thin walls of arbitrary conductivity parallel to the field, and (ii) non-conducting walls parallel to the field and thin walls of arbitrary conductivity perpendicular to the field. Sezgin (1987) has developed an analytical solution for the velocity field and magnetic field for the MHD flow of an incompressible, viscous and electrically conducting fluid in a rectangular duct, by reducing the problem to the solution of a Fredholm integral equation of the second kind, which has been solved numerically. Sai and Rao (2000) examined the effects of suction or injection on an incompressible laminar flow in a rectangular duct with non-conducting walls in the presence of an imposed transverse magnetic field. Sloan and Smith (1966) presented an exact solution for the steady laminar flow of a viscous, incompressible, electrically conducting liquid in a rectangular pipe between conducting plates under the influence of an imposed transverse magnetic field. Yakhot et al (1999) numerically solved the problem of pulsating laminar flow of a viscous, incompressible liquid in a rectangular duct.

The Hall and ion slip terms in Ohm's law were ignored in most of the MHD flow problems. However, in the presence of strong magnetic field, the influence of Hall current and ion slip are important. Tani (1962) studied the Hall effects on the steady motion of electrically conducting viscous fluid in channels. Hall and ion slip effects on MHD Couette flow with heat transfer have been considered by Soundelgekar e. al. (1979). Many of the problems in the literature deal with MHD flow between parallel plates/flow through circular pipes with Hall and ion slip effects, but not much attention has been given to the flow through a closed rectangular channel. Sayed and Attia (2005) have considered the Hall effects on

Paper accepted March, 2008. Technical Editor: Francisco Ricardo Cunha.
MHD flow and heat transfer through a rectangular duct for the case of a viscous incompressible electrically conducting Bingham fluid.

It is known that many of the industrially and technologically important fluids are electrically conducting fluids and behave like a non-Newtonian fluid. The theory of micropolar fluids initiated by Eringen (1966) exhibits some microscopic effects arising from the local structure and micro motion of the fluid elements. Further, they can sustain couple stresses and include classical Newtonian fluid as a special case. The model of micropolar fluid represents fluids consisting of rigid randomly oriented (or spherical) particles suspended in a viscous medium where the deformation of the particles is ignored. The fluids containing certain additives, some polymeric fluids and animal blood are examples of micropolar fluids. The mathematical theory of equations of micropolar fluids and applications of these fluids in the theory of lubrication and in the theory of porous media is presented by Lukaszewicz (1999).

In this paper, the effects of Hall and ion-slip are studied when an electrically conducting micropolar fluid flows in a rectangular channel in the presence of an imposed transverse magnetic field. The governing equations are solved numerically using finite differences.

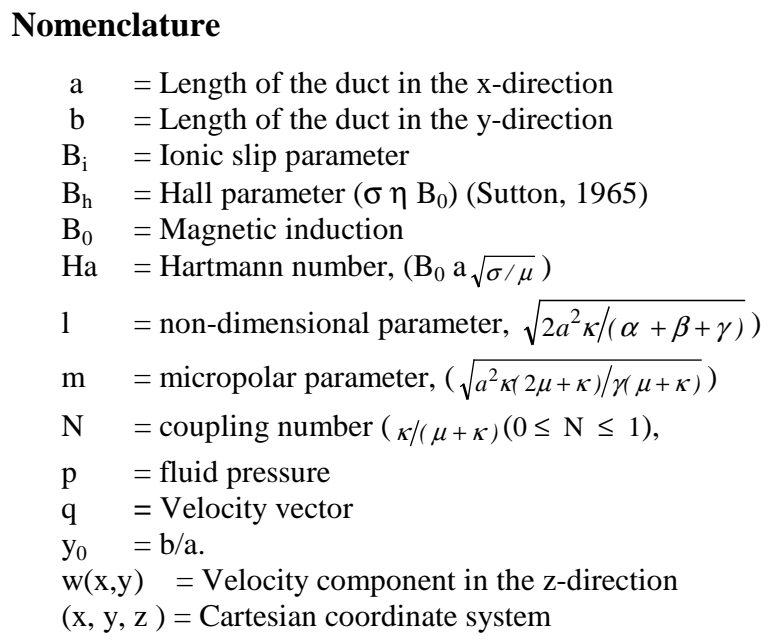

\section{Greek Symbols}

$\alpha \quad=$ material constant (viscosity coefficient) 


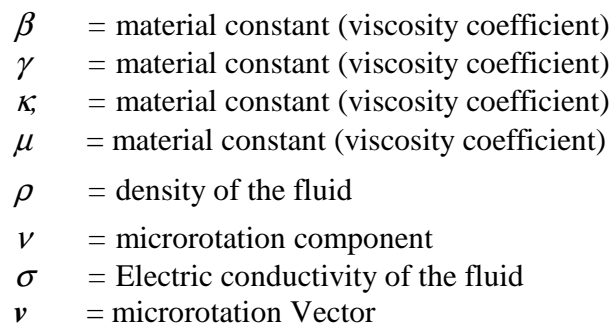

\section{Subscripts}

$i \quad$ relative to ion slip parameter

$\mathrm{h}$ relative to hall parameter

o relative to magnetic induction

1 relative to component in $\mathrm{x}$-direction

2 relative to component in $\mathrm{y}$-direction

\section{Formulation of the Problem}

Consider the steady flow of incompressible micropolar fluid through a rectangular duct with uniform cross-section. Choose the Cartesian coordinate system such that the $\mathrm{z}$-axis along the axis of the duct and $x, y$-axes along the sides and the origin at the center of the cross-sectional rectangle. Let ' $a$ ' and ' $b$ ' be the lengths of the duct in $\mathrm{x}$ and $\mathrm{y}$ directions respectively. The flow is subjected to a uniform magnetic field that can be directed arbitrary in a plane perpendicular to the z-axis. Assume that the flow is steady and magnetic Reynolds number is very small so that the induced the magnetic field can be neglected. The electron-atom collision frequency is assumed to be relatively high, so that the Hall effect and the ion slip cannot be neglected. The velocity vector of the fluid is given as $\boldsymbol{q}=w(x, y) \hat{k}$ and the microrotation vector as $\boldsymbol{v}=v_{1}(x, y) \hat{i}+v_{2}(x, y) \hat{j}$. Within the framework of these assumptions, the equations that govern the MHD flow of micropolar fluid in the absence of both body force and body couple are

$$
\begin{aligned}
& -\frac{\partial p}{\partial z}+\kappa\left(\frac{\partial v_{2}}{\partial x}-\frac{\partial v_{1}}{\partial y}\right)+(\mu+\kappa)\left(\frac{\partial^{2} w}{\partial x^{2}}+\frac{\partial^{2} w}{\partial y^{2}}\right)-\left\{\frac{\sigma B_{0}^{2}\left(1+B_{i} B_{h}\right)}{\left.\left(1+B_{i} B_{h}\right)^{2}+B_{h}^{2}\right\}}\right\}^{w=0} \\
& -2 \kappa y+\kappa \frac{\partial w}{\partial y}-\gamma \frac{\partial}{\partial y}\left(\frac{\partial v_{2}}{\partial x}-\frac{\partial v_{1}}{\partial y}\right)+(\alpha+\beta+\gamma) \frac{\partial}{\partial x}\left(\frac{\partial v_{1}}{\partial x}+\frac{\partial v_{2}}{\partial y}\right)=0 \\
& -2 \kappa \vartheta_{2}-\kappa \frac{\partial w}{\partial x}+\gamma \frac{\partial}{\partial x}\left(\frac{\partial v_{2}}{\partial x}-\frac{\partial v_{1}}{\partial y}\right)+(\alpha+\beta+\gamma) \frac{\partial}{\partial y}\left(\frac{\partial v_{1}}{\partial x}+\frac{\partial v_{2}}{\partial y}\right)=0
\end{aligned}
$$

In the present problem flow variables are invariant in the flow direction (z-direction), except the pressure gradient $\partial p / \partial z$, which is a constant. The material constants $\mu, \kappa, \alpha, \beta$ and $\gamma$ satisfy the following inequalities (Eringen,1966).

$$
\kappa \geq 0,2 \mu+\kappa \geq 0,3 \alpha+\beta+\gamma \geq 0, \quad \gamma \geq|\beta|
$$

Introducing the following non-dimensional variables

$x=a \tilde{x}, y=a \tilde{y}, w=\frac{p_{0} a^{2}}{\mu} \tilde{w}, v_{1}=\frac{p_{0} a}{\mu} \tilde{v}_{1}, v_{2}=\frac{p_{0} a}{\mu} \tilde{v}_{2}$

where $p_{0}=\partial p / \partial z$, into the equations (1) - (3) and dropping tildes, we get

$$
\begin{gathered}
-1+\frac{N}{1-N}\left(\frac{\partial v_{2}}{\partial x}-\frac{\partial v_{1}}{\partial y}\right)+\frac{1}{1-N}\left(\frac{\partial^{2} w}{\partial x^{2}}+\frac{\partial^{2} w}{\partial y^{2}}\right)-\frac{\left(1+B_{i} B_{h}\right) H a^{2}}{\left.\left(1+B_{i} B_{h}\right)^{2}+B_{h}^{2}\right\}^{w=0}} \\
v_{1}=\frac{1}{2} \frac{\partial w}{\partial y}-\frac{2-N}{m^{2}} \frac{\partial}{\partial y}\left(\frac{\partial v_{2}}{\partial x}-\frac{\partial v_{1}}{\partial y}\right)+\frac{1}{l^{2}} \frac{\partial}{\partial x}\left(\frac{\partial v_{1}}{\partial x}+\frac{\partial v_{2}}{\partial y}\right)=0 \\
v_{2}=\frac{1}{2} \frac{\partial w}{\partial x}+\frac{2-N}{m^{2}} \frac{\partial}{\partial x}\left(\frac{\partial v_{2}}{\partial x}-\frac{\partial v_{1}}{\partial y}\right)+\frac{1}{l^{2}} \frac{\partial}{\partial y}\left(\frac{\partial v_{1}}{\partial x}+\frac{\partial v_{2}}{\partial y}\right)=0
\end{gathered}
$$

where

$$
\begin{aligned}
& l^{2}=\frac{2 a^{2} \kappa}{\alpha+\beta+\gamma} \\
& N=\frac{\kappa}{\mu+\kappa} \text { coupling number }(0 \leq N \leq 1), \\
& m^{2}=\frac{a^{2} \kappa(2 \mu+\kappa)}{\gamma(\mu+\kappa)} \text { micropolar parameter, } \\
& H a=B_{0} a \sqrt{\sigma / \mu} \text { Hartmann number }
\end{aligned}
$$

The boundary conditions are usual no-slip and hyper stick conditions given by

$$
\begin{aligned}
& w=0 \text { at } x= \pm 1 \text { and } y= \pm y_{0} \\
& v_{1}=v_{2}=0 \text { at } x= \pm 1 \text { and } y= \pm y_{0}
\end{aligned}
$$

The governing equations given by Eq.(6), (7) and (8) along with the boundary conditions Eq.(9) are solved numerically using Finite Difference Method. The derivatives are replaced by the central difference approximations and using Gauss-Seidel iteration method, the resulting algebraic system of equations is solved. A numerical experiment was conducted with various meshes in the rectangular region and axial step lengths in $\mathrm{X}$ and $\mathrm{Y}$ directions to check the independence of the mesh resolution of the numerical results. There are three mesh distributions tested in the analysis. They are $21 \times 21$, $41 \times 41$ and $81 \times 81$ respectively. It is found that the deviations in the velocity and microrotation components calculated with $41 \times 41$ and $81 \times 81$ are always less than $10^{-6}$. Therefore, the computations with mesh distribution of $41 \times 41$ are considered to be sufficiently accurate to describe the flow in this study.

\section{Results and Discussion}

To explicitly see the effects of various parameters like coupling number $(\mathrm{N})$, micropolar fluid parameter $(m)$, magnetic parameter $(H a)$, Hall parameter $\left(B_{h}\right)$ and ion slip parameter $\left(B_{i}\right)$ on velocity and microrotation components, these quantities are numerically evaluated by dividing the rectangular region into a grid of mesh points $\left(\mathrm{x}_{\mathrm{i}}, \mathrm{y}_{\mathrm{j}}\right)$ and the results are graphically presented.

Figure 1 shows the profiles of velocity $(w)$ and microrotation components $\left(v_{1}\right.$ and $\left.v_{2}\right)$ for $y_{0}=1, B_{i}=2, B_{h}=2, H a=2, m=5, l=1.0$. It can be observed that the flow is symmetric in the $\mathrm{x}$ and $\mathrm{y}$ directions and as it is expected the velocity is maximum at the center of the rectangle.

The microrotation components $v_{l}$ and $v_{2}$ (Figs.1 (b) and (c)) are symmetric about the $\mathrm{x}$ and $\mathrm{y}$-axis respectively and they are zero at the center of the rectangle (Eringen, 1966). 


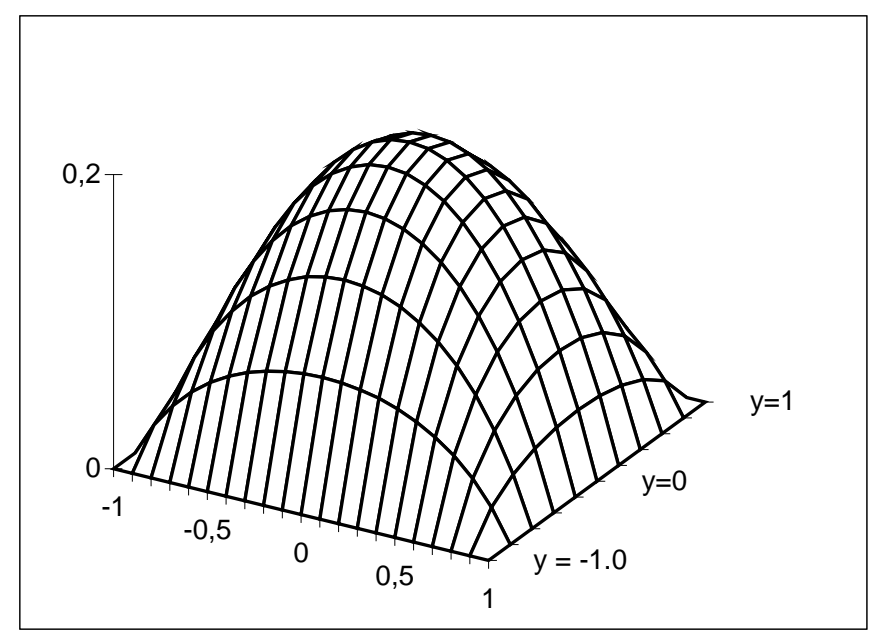

(a) Profile of velocity component (w)

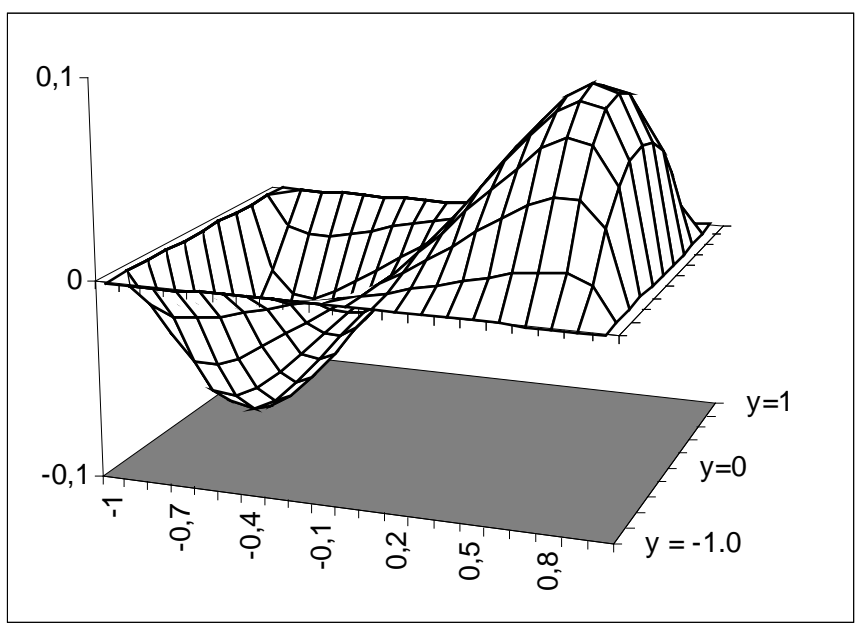

(b) Profile microrotation component $\left(v_{1}\right)$

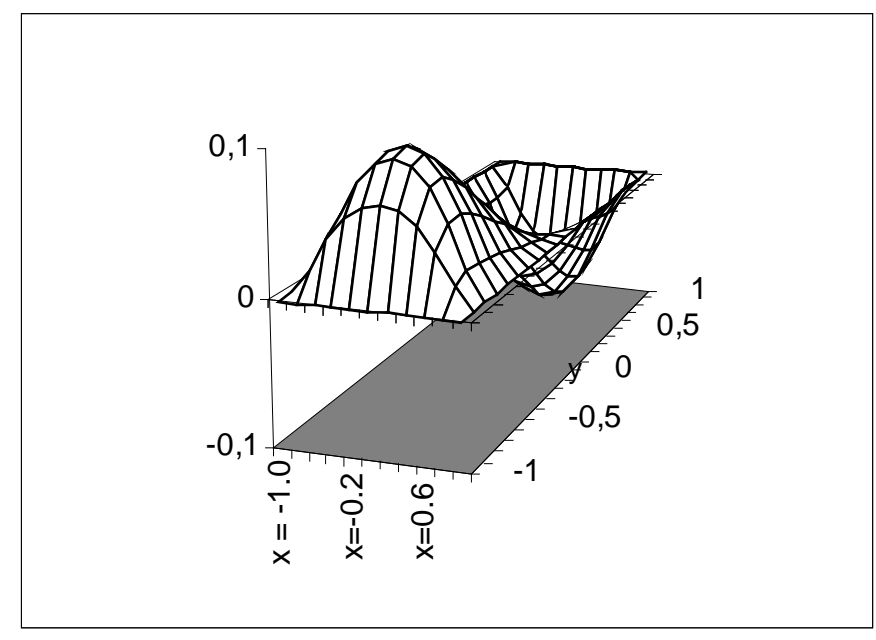

(c) Profile microrotation component $\left(v_{2}\right)$

Figure 1. Profiles of velocity $(w)$ and microrotation components ( $v_{1}$ and $v_{2}$ ) for $\mathrm{y}_{0}=1, \mathrm{~B}_{\mathrm{i}}=2, \mathrm{~N}=0.4, \mathrm{~B}_{\mathrm{h}}=2, \mathrm{Ha}=2, \mathrm{~m}=5, \mathrm{I}=1.0$.
The variation of velocity and micro rotation components with $\mathrm{x}$ at the centerline of the channel (i.e. $y=0$ ) for different values of the coupling number $(\mathrm{N})$ and for $y_{0}=1, B_{h}=2, B_{i}=2, H a=2, l=1.0$, and $m=5$ is shown in Figs. 2 (a) $-2(\mathrm{c})$.

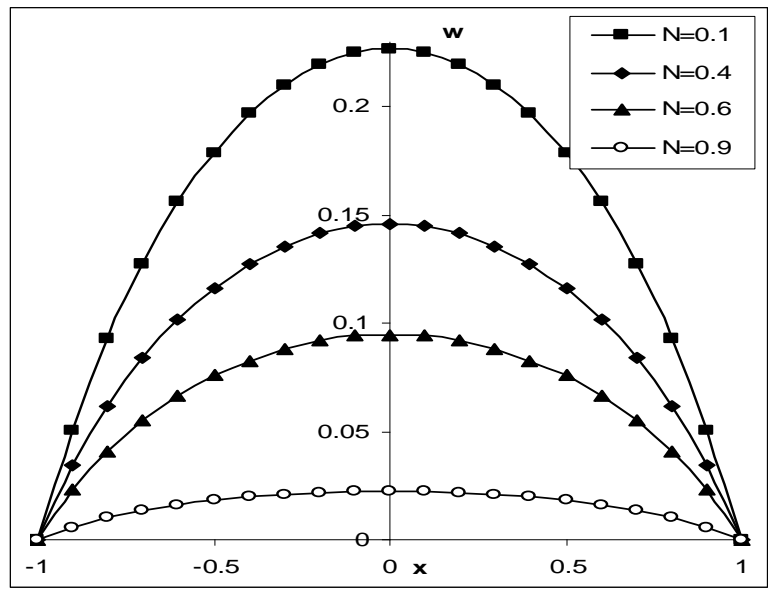

(a) The effect of $\mathrm{N}$ on velocity component (w)

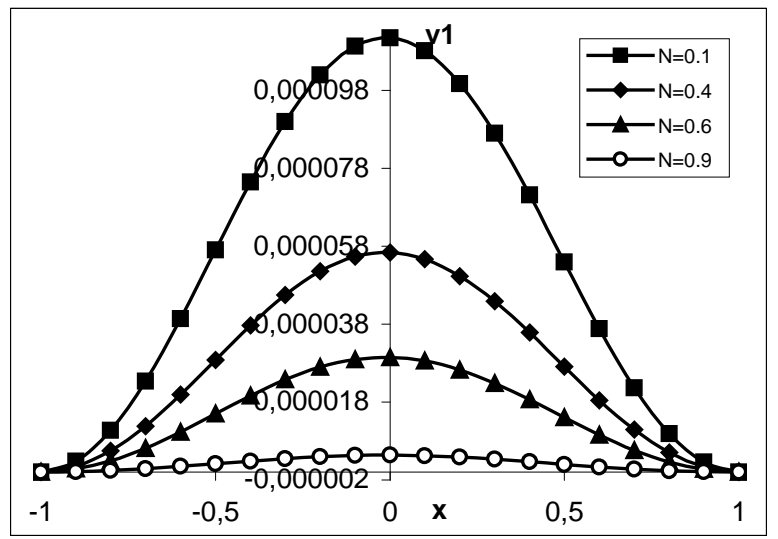

(b) The effect of $\mathbf{N}$ on microrotation component $\left(v_{1}\right)$

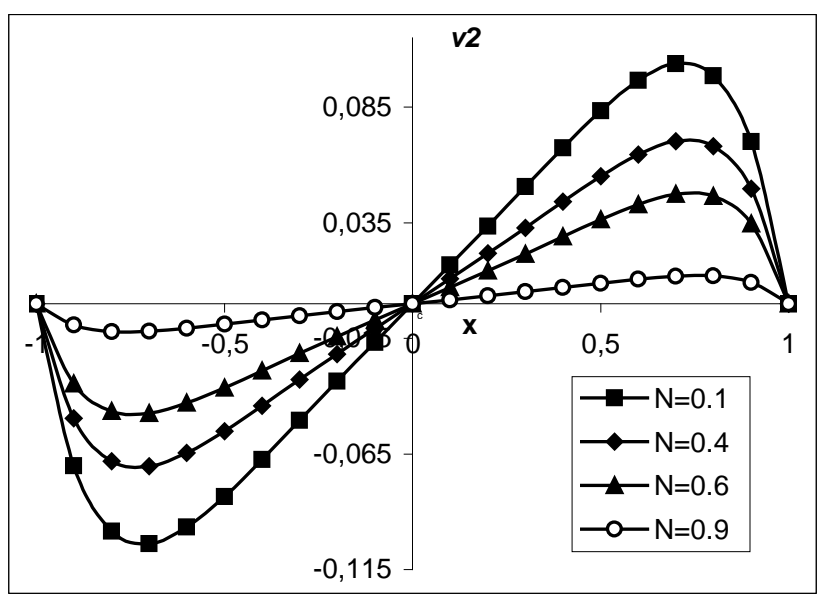

(c) The effect of $\mathrm{N}$ on microrotation component $\left(\mathrm{v}_{2}\right)$

Figure 2. The effect of $\mathrm{N}$ on velocity component $(\mathrm{w})$ and on microrotation components $\left(v_{1}\right.$ and $v_{2}$ ) for $\mathrm{y}=0, \mathrm{y}_{0}=1, \mathrm{~B}_{\mathrm{i}}=2, \mathrm{~B}_{\mathrm{h}}=2, \mathrm{Ha}=2, \mathrm{~m}=5, \mathrm{l}=1.0$. 
The velocity $w$ and microrotation component $v_{l}$ are decreasing as the coupling number increases. In the limit $N \rightarrow 0$, equations (6) and (7) reduce to the corresponding equations for a viscous fluid. Hence, it can be noticed that the velocity in the case of micropolar fluid is less than that of viscous fluid. The microrotation component $v_{2}$ increases near to one side of the rectangle and decreases near to the other side of the rectangle symmetrically with increasing $\mathrm{N}$, showing a reverse rotation near the boundaries. Further, it can be observed that for fixed $\mathrm{N}$, the microrotation components increase symmetrically with increasing y except at the region near the sides of the rectangle where it shows decreases.

Figures 3(a) - 3(c) analyze the effect of $m$ on the velocity and microrotation for $B_{h}=2, B_{i}=2, H a=2, l=1.0$ and $N=0.2$. It can be observed that as the value of $m$ increases the velocity decreases and micrortation component $v_{l}$ increases. The microrotation component $v_{2}$ increases near to one side of the rectangle and decreases near to the other side of the rectangle symmetrically with increasing micropolar parameter $(\mathrm{m})$.

The effect of magnetic parameter $(\mathrm{Ha})$ on the velocity and micro rotation is shown in Figs. 4(a) - 4(c). It can be seen from these figures that the velocity component, as it is expected, becomes small for large values of $\mathrm{Ha}$. This happens because of the imposing of a magnetic field normal to the flow direction. This magnetic field gives rise to a resistive force and slows down the movement of the fluid. Similarly the microrotation component $v_{I}$ decreases and $v_{2}$ increases near to one side of the rectangle and decreases near to the other side of the rectangle symmetrically with increasing magnetic parameter.

Figures 5(a) - 5(c) show the velocity and microrotation profiles for different values of ionic slip parameter $\left(B_{i}\right)$. The velocity component and microrotation component $v_{l}$ increase as the ionic slip parameter increases and the microrotation $v_{2}$ components increase near to one side of the rectangle and decrease near to the other side of the rectangle symmetrically with $B_{i}$ increase. As $B_{i}$ increases, the effective conductivity also increases, in turn, decreases the damping force on velocity, and hence the velocity increases.

Figures 6(a) - 6(c) show the effect of Hall parameter $\left(B_{h}\right)$ on velocity and micro rotation. It can be seen from these figures that the Hall parameter increases the velocity and microrotation component $v_{l}$. Also microrotation component $v_{2}$ increases in magnitude as $B_{h}$ increases. Inclusion of Hall parameter decreases the resistive force imposed by the magnetic field due to its effect in reducing the effective conductivity.

\section{Conclusions}

The steady conducting micropolar fluid through a rectangular duct under the influence of an applied uniform magnetic field has been studied, considering Hall and ionic slip effects. The governing equations of micropolar fluid are solved numerically using finite difference method. The effects of magnetic field, the Hall parameter, the Ion-slip parameter and Hartman number on the velocity and micro rotation components have been investigated. It is shown that increasing the magnetic field decreases the velocity and the micro rotation, while increasing the Hall and ion-slip parameter there is an increase in velocity and also the micro rotation increases in magnitude.

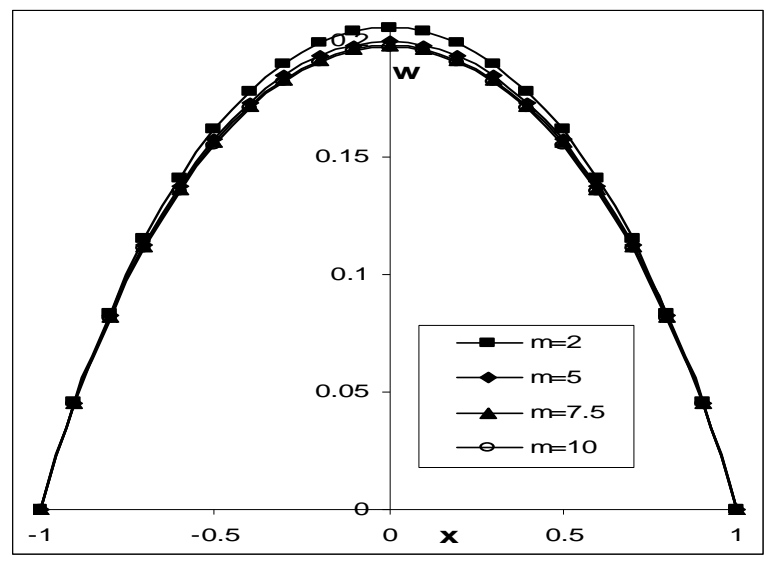

(a) The effect of $m$ on velocity component (w)

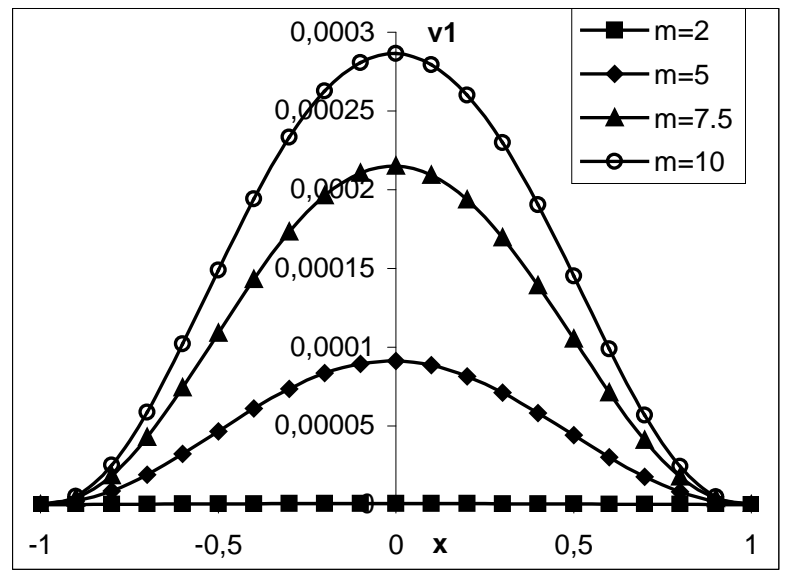

(b) The effect of $\mathrm{m}$ on microrotation component $\left(\mathbf{v}_{1}\right)$

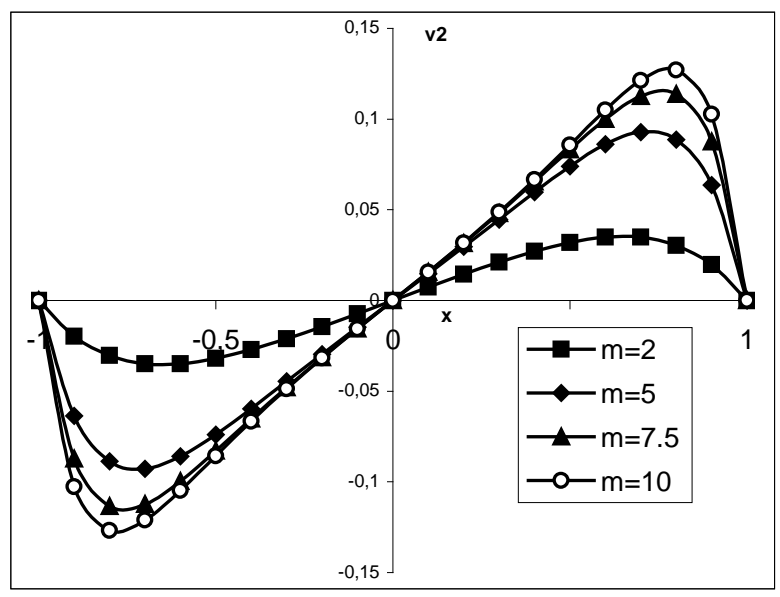

(c) The effect of $\mathbf{m}$ on microrotation component $\left(v_{2}\right)$

Figure 3. The effect of $m$ on velocity component $(w)$ and on microrotation components $\left(v_{1}\right.$ and $\left.v_{2}\right)$ for $\mathrm{y}=0, \mathrm{y}_{0}=1, \mathrm{Bi}=2, \mathrm{~B}_{\mathrm{h}}=2, \mathrm{Ha}=2, \mathrm{~N}=0.2, \mathrm{I}=1.0$. 


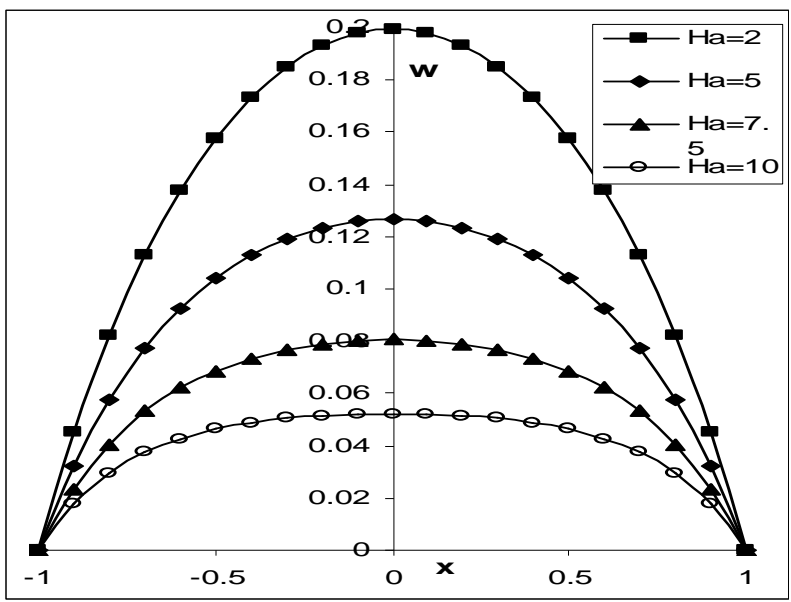

(a) The effect of $\mathrm{H}_{a}$ on velocity component (w)

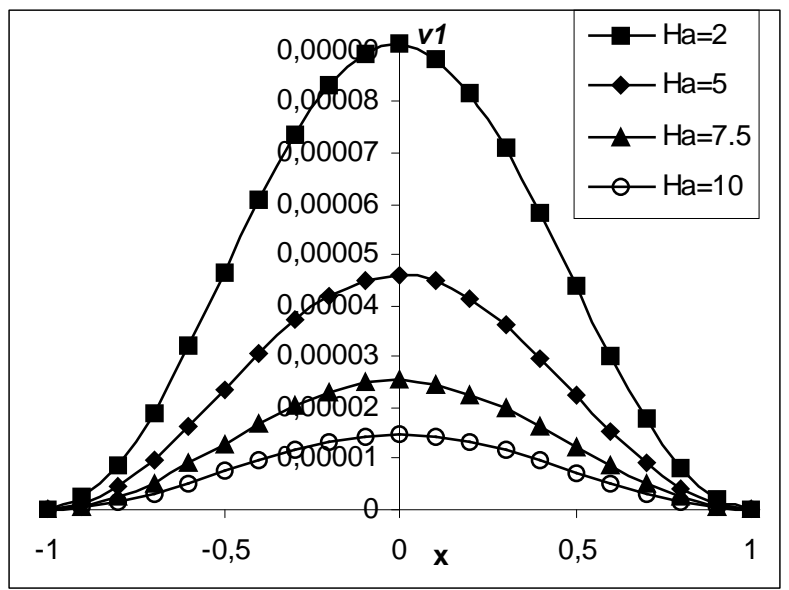

(b) The effect of $\mathrm{H}_{\mathrm{a}}$ on microrotation component $\left(\mathrm{v}_{1}\right)$

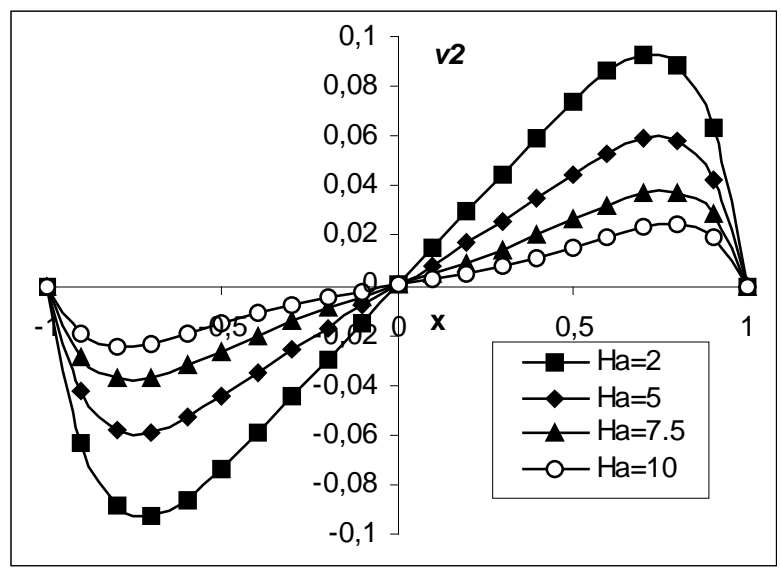

(c) The effect of $\mathrm{H}_{\mathrm{a}}$ on microrotation component $\left(\mathrm{v}_{2}\right)$

Figure 4. The effect of $\mathrm{H}_{\mathrm{a}}$ on velocity component (w) and on microrotation components $\left(v_{1}\right.$ and $\left.v_{2}\right)$ for $\mathrm{y}=0, \mathrm{y}_{0}=1, \mathrm{Bi}=2, \mathrm{~B}_{\mathrm{h}}=2, \mathrm{~m}=5, \mathrm{~N}=0.2, \mathrm{l}=1.0$.

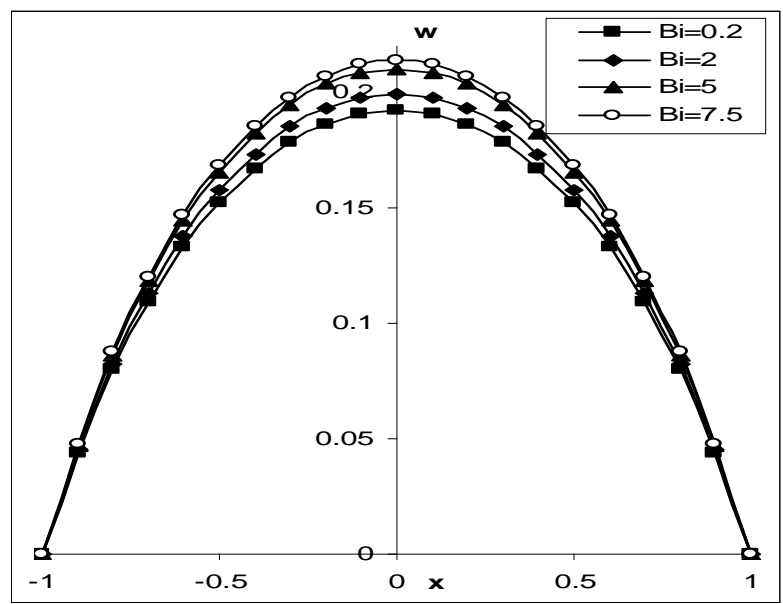

(a) The effect of $B_{i}$ on velocity component (w)

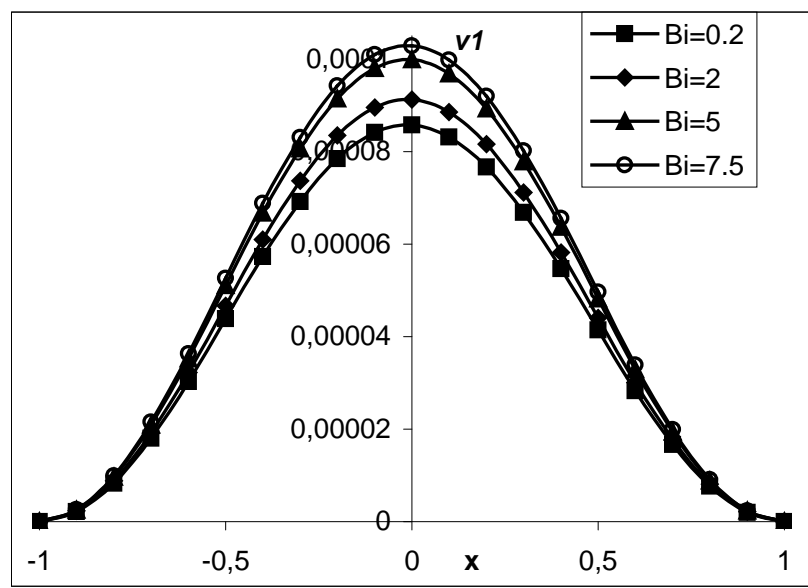

(b) The effect of $B_{i}$ on microrotation component $\left(v_{1}\right)$

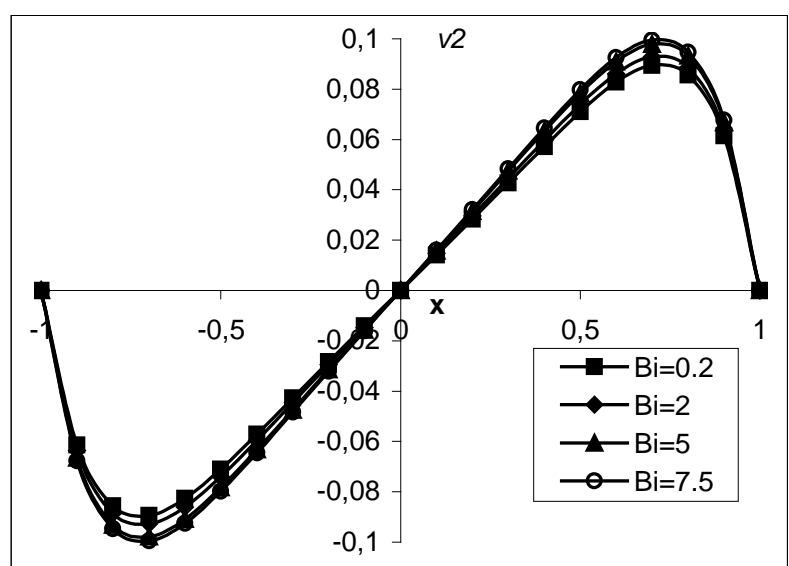

(c) The effect of $B_{i}$ on microrotation component $\left(v_{2}\right)$

Figure 5. The effect of $B_{i}$ velocity component $(w)$ and on microrotation components $\left(v_{1}\right.$ and $\left.v_{2}\right)$ for $\mathrm{y}=0, \mathrm{y}_{0}=1, \mathrm{H}_{\mathrm{a}}=2, \mathrm{~B}_{\mathrm{h}}=2, \mathrm{~m}=5, \mathrm{~N}=0.2, \mathrm{l}=1.0$. 


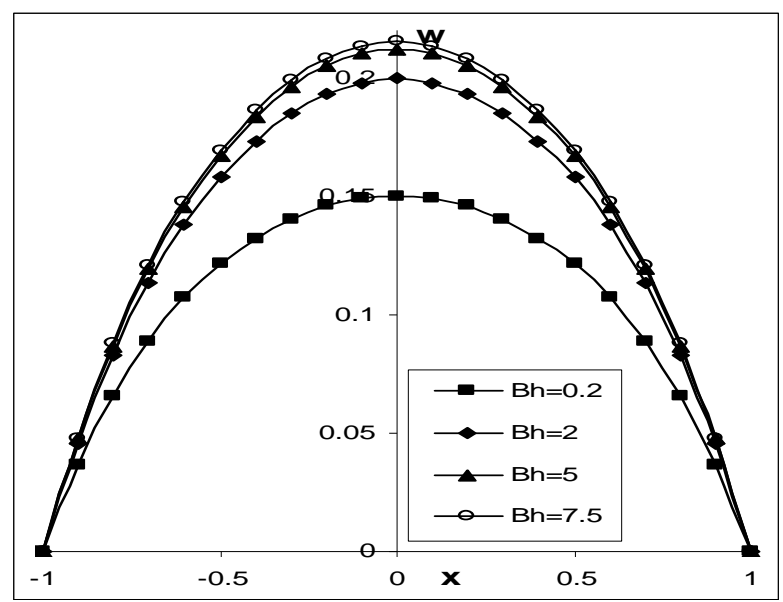

(a) The effect of $B_{h}$ on velocity component (w)

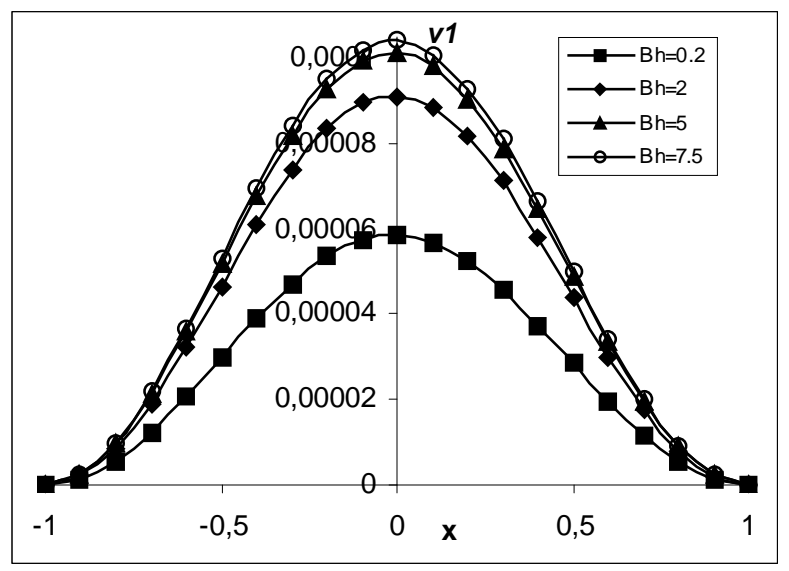

(b) The effect of $B_{h}$ on microrotation component $\left(v_{1}\right)$

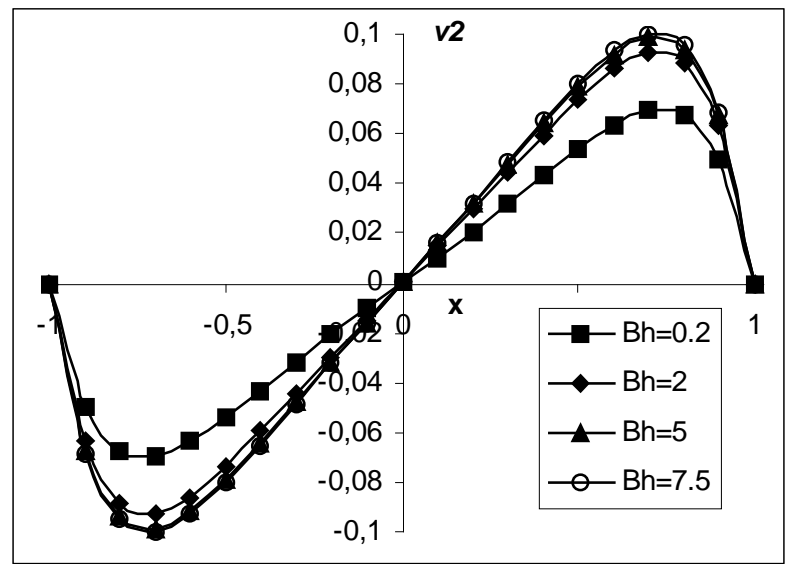

(c) The effect of $B_{h}$ on microrotation component $\left(v_{2}\right)$

Figure 6. The effect of $B_{h}$ velocity component $(w)$ and on microrotation components $\left(v_{1}\right.$ and $v_{2}$ ) for $\mathrm{y}=0, \mathrm{y}_{0}=1, \mathrm{H}_{\mathrm{a}}=2, \mathrm{~B}_{\mathrm{i}}=2, \mathrm{~m}=2, \mathrm{~N}=0.2, \mathrm{l}=1.0$.

\section{References}

Eringen, A. C., 1966, "The theory of micropolar fluids", J. Math. Mech., vol. 16, pp 1-16.

Lukaszewicz, G, 1999, "micropolar fluids - theory and applications", Birkhauser, Boston.

Hunt J. C. R., 1965, "Magnetohydrodynamic flow in a rectangular duct". J. Fluid Mech., Vol. 21, pp. 577-590.

Sai, K. S. and Nageswara Rao, B., 2000, "Magnetohydrodynamic flow in a rectangular duct with suction and injection", Acta Mech., Vol. 140, pp.(1-2).

Sayed-Ahmed, M.E. and Attia, H.A., 2005, "The effect of Hall current on magneto hydrodynamic flow and heat transfer for Bingham fluids in a rectangular duct", Canadian Journal of Physics, Vol. 83 No.6, pp. 637-651.

Sezgin, M.,1987, "Magnetohydrodynamic flow in a rectangular duct", Int. J. Num. Meth. Fluids, Vol 7 (7), pp. $697-718$.

Sloan, D. M. and Smith, P., 1966, "Magnetohydrodynamic Flow in a Rectangular Pipe between Conducting Plates", ZAMM, Vol. 46, no. 7, pp. 409-484.

Soundalgekar, V. M., Vighnesam, N. V. and Takhar, H. S., 1979, "Hall and ion-slip effects in MHD Coutte flow with heat transfer", IEEE Transactions on Plasma Sci. vol.7, pp.178-182.

Sutton,G.W. and Sherman, A., 1965, "Engineering Magnetohydrodynamics", McGraw-Hill, New York.

Tani. I., 1962. "Steady flow of conducting fluids in channels under transverse magnetic fields, with consideration of Hall effects", $J$. of Aerospace Scie., vol. 29, pp. 297-305.

Yakhot A., Arad M, and Ben-Dor G.,1999 "Numerical investigation of a laminar pulsating flow in a rectangular duct", Int. J. num. meth. fluids, Vol. 29 No.8, pp. 935-950. 